\title{
Credit Rating Modelled with Reflected Stochastic Differential Equations
}

\author{
Adeyemi Adewale Sonubi \\ Federal University of Agriculture, Abeokuta, Nigeria \\ Email: sonubi03@yahoo.com
}

Received 30 July 2014; revised 13 September 2014; accepted 28 September 2014

Copyright (C) 2014 by author and Scientific Research Publishing Inc.

This work is licensed under the Creative Commons Attribution International License (CC BY). http://creativecommons.org/licenses/by/4.0/

c) (i) Open Access

\begin{abstract}
This research paper is focused on the modelling of credit rating, using reduced form approach, in which intensity is defined endogenously based on the firm's cashflow. It was modelled with reflected stochastic differential equation; this was adopted to evaluate the credit rating of a firm where the reflection function $\phi(t)$ (i.e. Brownian local time) was used to detect default and measure time spent at default. Through this, the credit rating is estimated within [0,1]; where " 0 " is the state of default and " 1 " is interpreted as undefaultable within a time interval $t \geq[0, \infty)$ under consideration.
\end{abstract}

\section{Keywords}

Brownian Local Time, Credit Rating, Cashflow, Default, Reflected Stochastic Differential Equation, Undefaultable

\section{Introduction}

The mathematical modelling of stock market using Brownian motion according to Protter [1] and Hobson [2] was first introduced in 1900 by L. Bachelier in his Ph.D thesis where he modelled the flunctuation of assets prices and the price derivatives. Protter in [1] claimed that Bachelier's work was not recognised but was rediscovered by L. Savage in the 1950s. He later alerted P. Samuelson [3] who suggested the use of geometric Brownian motion to model stock prices.

A major breakthrough occurred in 1973 when Black and Scholes [4] proposed a method to price European options via an explicit formula by making use of Itǒ stochastic calculus and the Markov property for diffusion. In the same year, Merton [5] with some modifications showed that the same analysis could be applied to American options on non-dividend paying common stocks. In the last two decades, the idea of using stochastic 
calculus for modelling prices of risky assets has generally been accepted and this has interestingly led to a new branch of applied probability theory called Mathematical Finance.

One of the most recent research areas in mathematical finance is the modeling of credit risk. Until recently, obligors were either classified to be of "good credit" and assumed not to default in payment of debt, or said to be of "bad credit" and failure to fulfill financial obligation was assumed. Probability of default was only introduced in the 1990s and financial institutions such as banks started to manage their credit portfolio using credit ratings.

M. Jeanblanc in [6] had earlier modelled default risk by providing a detailed analysis of a case when the flow of information available to an agent reduced to the observations of the random time which modelled the default event. In the research work, a restricted condition was posed in the model by assuming an hypothesis which postulated the invariance of the martingale property with respect to the enlargement of the Brownian filtration by the observation of a default time. But in this paper, instead of such hypothesis, reflection of the boundary of a stochastic differential equation shall be used to model default.

The main focus of this paper is to model credit rating using one-dimensional reflected stochastic differential equation with motivation from A. V. Skorohod [7], H. Tananka [8], and N. Ikeda and S. Watanabe [9] [10]. This would be done through incomplete information model which was introduced by Duffie and Lando [11] and Giesecke [12]. It has been analysed by Giesecke [12], Jarrow and Protter [13] amongst others that this model bridges the gap between the structural approach and the reduced form approach in modelling credit risk by the assumption of the completeness of information available to the modeler. This eventually makes a structural model whose default time is predictable to be transformed into a reduced form model whose default is totally a surprise. Based on this incomplete information about the firms asset or default barrier, the structural model becomes a reduced form model whose intensity of default is no more exogenously defined but now determined endogenously within the model.

\section{Preliminaries}

Definition 2.1. Let $(\Omega, \mathcal{G}, \mathbb{P})$ be a probability space, a random variable $\tau: \Omega \rightarrow[0, \infty]$ is called a $\mathcal{G}_{t}{ }^{-}$ stopping time if $\{\omega: \tau(\omega) \leq t\} \in \mathcal{G}_{t}$ for any $t \in[0, \infty)$. The stopping time $\tau$ is said to be predictable if there exists a non-decreasing sequence of stopping times $\left(S_{i}\right)_{i \geq 1}$ with $\kappa=\bigcap_{i=1}^{\infty}\left\{S_{i}<\tau\right\}$, then

$$
\mathbb{P}\left(\left\{\omega \in \Omega: \lim _{i \rightarrow \infty} S_{i}=\tau\right\} \cap \kappa\right)=1 .
$$

The stopping time $\tau$ is said to be totally inaccessible if it comes with no advance warning (i.e. a complete surprise). It can be defined as

$$
\mathbb{P}\left(\left\{\omega \in \Omega: \lim _{i \rightarrow \infty} S_{i}=\tau\right\} \cap \kappa\right)=0
$$

where $\left(S_{i}\right)_{i \geq 1}$ is a non-decreasing sequence of stopping times as defined above.

Theorem 2.1. A uniformly integrable process $N_{\tau}$ such that $\tau$ is a finite stopping time is a submartingale if and only if it has a decomposition

$$
N_{\tau}=M_{\tau}+A_{\tau}
$$

where $M_{\tau}$ is a uniformly integrable martingale and $A_{\tau}$ is a predictable increasing process, both are null at 0 .

Definition 2.2. A process $C_{t}$ is called the $\mathcal{G}_{t}$-compensator of the process $N_{t}$ if and only if the following conditions are satisfied:

i) $C_{t}$ is a $\mathcal{G}_{t}$-predictable increasing process, with $C_{0}=0$.

ii) The process $N_{t}-C_{t}$, called the compensated process, follows a $\mathcal{G}_{t}$-martingale.

Definition 2.3. Let $Y(t)$ be a one-dimensional Brownian motion defined on a probability space $(\Omega, \mathcal{G}, \mathbb{P})$. A local time which can also be called a sojourn time density of $Y(t)$ is a non-negative random variable $\phi(t, y)$ which satisfies the following properties:

i) $(t, y) \rightarrow \phi(t, y)$ is continuous.

ii) For every Borel subset $D$ of $\mathbb{R}$ and $t \geq 0$ 


$$
\int_{0}^{t} \mathbf{1}_{D}(Y(s)) \mathrm{d} s=2 \int_{D} \phi(t, y) \mathrm{d} y
$$

$\phi(t, y)$ is unique and can be written as

$$
\phi(t, y)=\lim _{\varepsilon \downarrow 0} \frac{1}{4 \varepsilon} \int_{0}^{t} \mathbf{1}_{(x-\varepsilon, x+\varepsilon)} Y(s) \mathrm{d} s .
$$

A local time is a process that describe the amount of time a diffusion process has spent at a given point therefore providing a description of the sample path of the process.

\section{Reflected Stochastic Differential Equations}

We shall consider a one-dimensional diffusion process $Y(t)$ on $\bar{D}=\mathbb{R}^{+}=\{y \geq 0\}$ which has only one boundary at $y=0$. We define $\bar{D}=\partial D \cup D$, where $\partial D=\{y \in \bar{D} ; y=0\}$ is the boundary of $\bar{D}$ and $D=\{y \in \bar{D} ; y>0\}$ is the interior of $\bar{D}$.

Let $\alpha(t, y): \bar{D} \rightarrow \mathbb{R}$ and $\beta(t, y): \bar{D} \rightarrow \mathbb{R} \times \mathbb{R}$ be continuous, a one dimensional reflected stochastic differential equation is as follows:

$$
\left\{\begin{array}{l}
\mathrm{d} Y(t)=\alpha(t, Y(t)) \mathrm{d} t+\beta(t, Y(t)) \mathrm{d} B(t)+\mathrm{d} \phi(t) ; \\
\mathbf{1}_{\{0\}}(Y(s)) \mathrm{d} s=\xi(Y(s)) \mathrm{d} \phi(s), \quad 0<s \leq t .
\end{array}\right.
$$

Remark 2.1. From [9] these equations can be intuitively interpreted as the following: The "reflection function" $\phi(t)$ is a non-decreasing process whose point of increase is only when $Y(t)$ is on the boundary $\partial D$ where it causes a reflection. It is called the local time of $Y(t)$ on $\partial D . B$ is defined as one-dimensional Brownian motion in the ordinary time such that $\mathrm{d}\langle B\rangle(t)=\mathrm{d} t$. The function $\xi(y)$ describes the rate of sojourn of $Y(t)$ on the boundary $\partial D$. When $\xi(y) \equiv 0$ then $\int \mathbf{1}_{\partial D}(Y(s)) \mathrm{d} s=0$ for $t \geq 0$ the boundary is said to be nonsticky and this also called instantaneous reflection. When $\xi(y)>0$ then $\int \mathbf{1}_{\partial D}(Y(s)) \mathrm{d} s>0$. The boundary is said to be sticky and this is also referred to as the delayed reflection.

Definition 2.4. Let $(\Omega, \mathcal{G}, \mathbb{P})$ be a probability space with a filtration $(\mathcal{G})_{t \geq 0}$. We call a family of stochastic process $X=(Y(t), B(t), \phi(t))$ a solution of (2.1) if the following holds:

i) $Y(t)$ is a $\bar{D}$-valued continuous $\left(\mathcal{G}_{t}\right)$-adapted process.

ii) $\phi(t)$ is a continuous $\left(\mathcal{G}_{t}\right)$-adapted increasing process such that $\phi(0)=0$ and $\int_{0}^{t} \mathbf{1}_{\partial D}(Y(s)) \mathrm{d} \phi(s)=\phi(t)$ for $t \geq 0$ a.s.

iii) There exists a one-dimensional Brownian motion $B(t)$ with $B(0)=0$ a.s.

iv) With probability one,

$$
Y(t)=Y(0)+\int_{0}^{t} \alpha(s, Y(s)) \mathrm{d} s+\int_{0}^{t} \beta(s, Y(s)) \mathrm{d} B(s)+\phi(t) .
$$

Theorem 2.2. Let $\alpha(t, y):[0, \infty) \times \mathbb{R} \rightarrow \mathbb{R}$ and $\beta(t, y):[0, \infty) \times \mathbb{R} \rightarrow \mathbb{R} \times \mathbb{R}$ be measurable functions that satisfy the linear growth and Lipschitz condition and let $\phi(t)$ be a continuous non-decreasing process, having point of increase only at the zeros of $Y(t)$, then there exist a unique $\mathbb{R}$-valued solution $Y(t)$ for the reflected stochastic differential equation

$$
\mathrm{d} Y(t)=\alpha(t, Y(t)) \mathrm{d} t+\beta(t, Y(t)) \mathrm{d} B(t)+\mathrm{d} \phi(t), \quad t \in[0, \infty) .
$$

Proof. See [8] and [10].

\section{Main Result}

Let $(\Omega, \mathcal{G}, \mathbb{P})$ be defined as a filtered complete probability space where $\mathcal{G}_{t}$ is the information set available to the manager of the firm to be rated. If $V_{t}$ is the net value of the firm at time $t>0$, then 


$$
\mathcal{G}_{t}=\sigma\left(V_{s} ; s \leq t\right) .
$$

Let another filtration $\mathcal{H}_{t}$ be defined which contains a reduced information set such as cash flow of the firm. This implies that $\mathcal{H}_{t} \subset \mathcal{G}_{t}$. The default time of the model with the filtration $\mathcal{H}_{t}$ becomes totally inaccessible stopping times because the information set has been reduced from the one being constantly monitored by the manager with predictable default time. Hence, it is a reduced form model with endogenously defined intensity on the firm's economic fundamentals which is cashflow.

Let us consider a one-dimensional reflected stochastic differential equation which shall be used to model credit rating of a firm. The model will assess the firm based on the cash flow over a period of time $t>0$, it will show the credit rating of such firm between the interval $[0,1]$ where point " 0 " means the firm is at a state of default which is the lowest rating and the point " 1 " means the firm is undefaultable which is the highest rating. The relevant barrier to be considered in this model is $\partial D=0$, where negative cashflow is still assumed to be at point zero.

$$
\left\{\begin{array}{l}
\mathrm{d} Y(t)=\alpha(t, Y(t)) \mathrm{d} t+\beta(t, Y(t)) \mathrm{d} B(t)+\mathrm{d} \phi(t) ; \\
\mathbf{1}_{\{0\}}(Y(s)) \mathrm{d} s=\xi(Y(s)) \mathrm{d} \phi(s) .
\end{array}\right.
$$

The model is made up of the following terms:

i) Let $Y(t)=$ the cashflow of the firm. This is made up of cash inflow (i.e. bank loans, interest on savings etc.) and cash outflow (i.e. purchase of stock, purchase of raw materials etc.). $Y(t)$ is a markov process since the present cashflow of the firm over a period of time $t>0$ will be used to determine the future credit rating of the firm without any consideration of the past cashflow.

ii) $\alpha(t, Y(t))=$ riskless cash, which represents the cash inflow.

iii) $\beta(t, Y(t))=$ risky cash, which represents the cash outflow.

iv) $\phi(t)=$ the reflection function that measures the time spent at default. It increases immediately the cashflow of the firm $Y(t)=0$. Where we define $\phi(t) \in(0,1)$ and $\phi(0)=0$.

v) $\xi(Y(t))=$ the default intensity.

vi) Let $R_{\phi(t)}$ be defined as the rating of a firm at time $t>0$. This a $\mathcal{H}$-martingale process since it is believed to be without any bias (i.e. it is "fair").

vii) $N_{\phi(t)}$ is default indicator process such that

$$
N_{\phi(t)}= \begin{cases}1 & \phi(t)>0 \\ 0 & \phi(t)=0\end{cases}
$$

viii) $C_{\phi(t)}$ act as the compensator which describes the cumulative default intensity and it is

$$
C_{\phi(t)}=\int_{0}^{t} \xi(Y(s)) \mathrm{d} \phi(s)=\int_{0}^{t} \mathbf{1}_{\{0\}}(Y(s)) \mathrm{d} s .
$$

Since the default indicator process $N_{\phi(t)}$ is a point process with one jump of size one at default, it is uniformly integrable, hence it is a submartigale. $C_{\phi(t)}$ is an increasing predictable process since $C_{\phi(t)}=\phi(t)$ when $\xi(Y(t))=1$ therefore by Doob-Meyer decomposition (Theorem 2.1), the credit rating of the firm will be equal

$$
R_{\phi(t)}=N_{\phi(t)}-C_{\phi(t)}
$$

Let us consider the following cases:

Case 1: Suppose throughout the period $t>0$ being considered, the cash inflow of the firm was always greater than the cash outflow (i.e. $Y(t)>0$ all through period $t>0$ ), this implies that throughout the period $t>0$ the firm did not default. Hence, the credit rating of such firm is $R_{\phi(t)}=1$.

Interpretation: This means the firm is termed Undefaultable throughout the period considered and such firm can be recommended to investors for future investment with very low risk.

Case 2: When the cash inflow is less than the cash outflow during the period of time $t>0$ being considered, default occurs. Now, suppose the firm's cashflow $Y(t)=0$ at time $s \in(0, t)$ but there was a recovery from such default within a short period (i.e. $Y(t)>0$ ), the default intensity is low. Hence the value of the cumulative rate of default is also very small say $\epsilon \in(0,0.5)$. The rating of the firm in this is the case is

$$
R_{\phi(t)}=1-\epsilon .
$$


Interpretation: Hence, such a firm will have high credit rating and the closer it is to 1 the higher the rating. Therefore investors could consider such firm as low risk firm and if given a loan the interest rate will be small.

Case 3: Suppose on the contrary, when the cashflow $Y(t)=0$, the firm tarried at this point for a very long period of time $k \in(0, t)$ or equivalently if the cumulative time of default within the period $(0, t)$ is considerably large. The cumulative rate of default will be equally large say $v \in[0.5,1)$. Therefore, the rating of the firm in this case is $R_{\phi(t)}=1-v$.

Interpretation: Such firm will be rated very low. Therefore investors could consider such a firm as a high risk firm. Granting a loan to such a firm will require a very high interest rate.

Case 4: Finally, suppose the cashflow of the firm is $Y(t)=0$ through out all the period $t>0$ being considered, then such firm has a credit rating is $R_{\phi(t)}=0$.

Interpretation: Such firm is said to be at a default state and the firm is of very high credit risk. Investors are advised to avoid investing in such firm until a credit migration occurs when the firm moves from such a default state to at least with low credit rating.

\section{Conclusions}

In conclusion, a firm's credit rating can be evaluated by investors in order to make decision on future investments and this has been done by modelling credit rating with reflected stochastic differential equation where the reflecting function detects the default time and it measures how long the firm stays at the point of default. Case 1 and Case 2 can be suggested as the optimal solution for investor to minimise risk.

Further research can be done through multidimensional extensions by the use of systems of reflected stochastic differential equations to analyse a set of ratings for various indexes in the risk management of the relevant stochastic financial models.

\section{References}

[1] Protter, P. (2001) A Partial Introduction to Financial Assest Pricing Theory. Stochastic Processes and Their Applications, 91, 169-203. http://dx.doi.org/10.1016/S0304-4149(00)00064-8

[2] Hobson, D. (2004) A Survey of Mathematical Finance. Proceedings of the Royal Society A, 460, 3369-3401. http://dx.doi.org/10.1098/rspa.2004.1386

[3] Samuelson, P. (1965) Rational Theory of Warrant Pricing. Industrial Management Review, 6, 13-31.

[4] Black, F. and Scholes, M. (1973) The Pricing of Options and Corporate Liabilities. Journal of Political Economy, 81, 637-659. http://dx.doi.org/10.1086/260062

[5] Merton, C.R. (1973) The Theory of Rational Option Pricing. Bell Journal of Economics and Management Science, 4, 141-183. http://dx.doi.org/10.2307/3003143

[6] Jeanblanc, M. and Rutkowski, M. (2000) Modelling of Default Risk: An Overview. Workshop Presentation, New York University, New York.

[7] Skorohod, A.V. (1961) Stochastic Equation for Diffusion Processes in a Bounded Region. Theory of Probability and Its Applications, 6, 264-274. http://dx.doi.org/10.1137/1106035

[8] Tanaka, H. (1979) Stochastic Differential Equations with Reflecting Boundary Condition in Convex Regions. Hiroshima Mathematical Journal, 9, 163-177.

[9] Ikeda, N. and Watanabe, S. (1989) Stochastic Differential Equation and Diffusion Process. 2nd Edition, North-Holland Publishing Company, Japan.

[10] Watanabe, S. (1971) On Stochastic Differential Equation for Multidimensional Diffusion Processes with Boundary Condition. Journal of Mathematics of Kyoto University, 11, 169-180.

[11] Duffie, D. and Lando, D. (2001) Term Structure of Credit Spreads with Incomplete Accounting Information. Econometrica, 69, 633-664. http://dx.doi.org/10.1111/1468-0262.00208

[12] Giesecke, K. (2006) Default and Information. Journal of Economics and Control, 30, 2281-2303. http://dx.doi.org/10.1016/j.jedc.2005.07.003

[13] Jarrow, R.A. and Protter, P. (2004) Structural versus Reduced form Models: A New Information Based Perspective. Journal of Investment Management, 2, 1-10. 
Scientific Research Publishing (SCIRP) is one of the largest Open Access journal publishers. It is currently publishing more than 200 open access, online, peer-reviewed journals covering a wide range of academic disciplines. SCIRP serves the worldwide academic communities and contributes to the progress and application of science with its publication.

Other selected journals from SCIRP are listed as below. Submit your manuscript to us via either submit@scirp.org or Online Submission Portal.
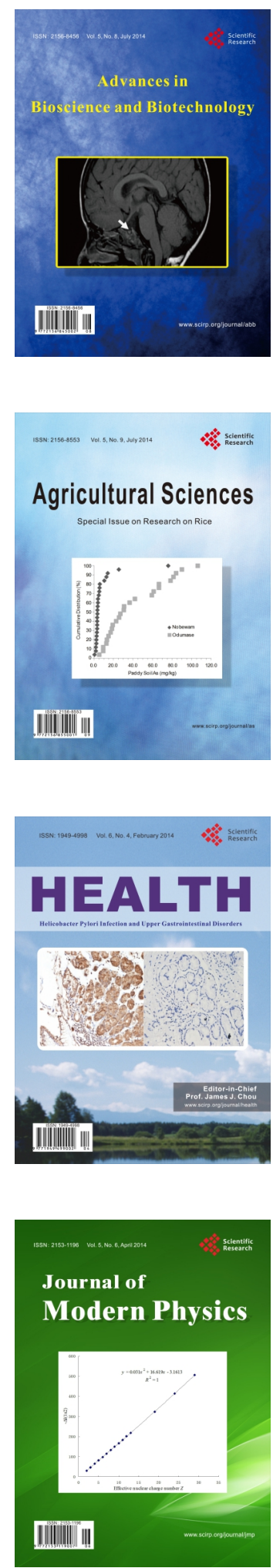
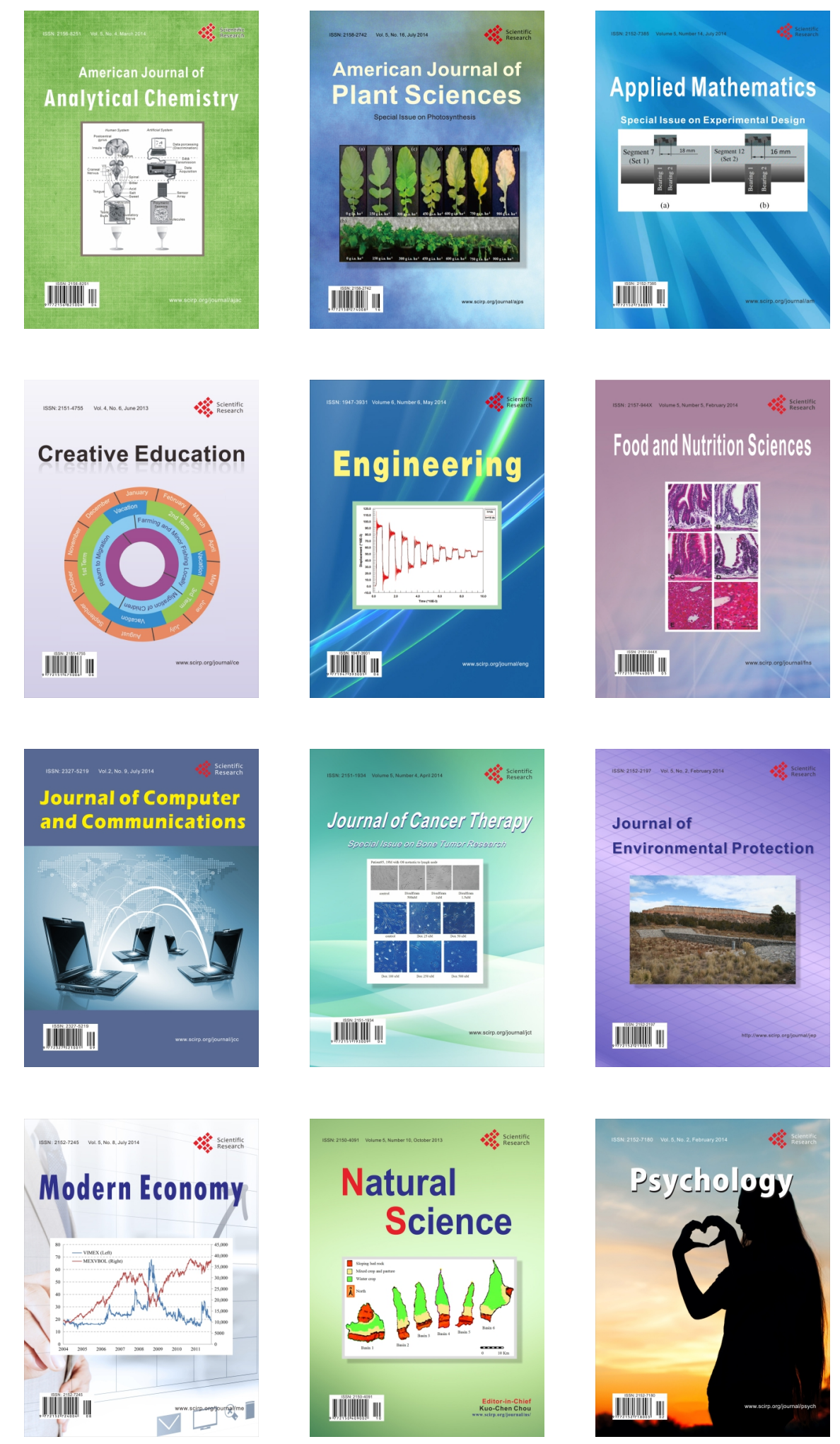